\title{
Variable Impedance Control of a Rehabilitation Robot for Modelling Physiotherapist's Motions ${ }^{\#}$
}

\author{
Barış Can Yalçın *, Erhan Akdoğan, Celal Sami Tüfekçi
}

\author{
Accepted $15^{\text {th }}$ August 2014
}

\begin{abstract}
This paper presents a variable impedance control method, which is used to teach a lower limb rehabilitation robot how to imitate exercise motions applied to a patient by a physiotherapist. To achieve this task, the characteristics of physiotherapist's motion are investigated. The proposed control method is based on estimating stiffness parameter of a physiotherapist's arm and generating impedance parameters of the robot to model the exercise motions. The effectiveness of proposed method is shown with simulation results.
\end{abstract}

Keywords: Rehabilitation, Impedance Control.

\section{Introduction}

The amount of people who are in need of being rehabilitated by a physiotherapist is increasing day by day. In order to address solutions for this necessity, robotic technologies in the field of rehabilitation have advanced considerably in an effort to satisfy expectations of patients who need physical therapy. In the last fifteen years, robotic systems for rehabilitation have been progressively investigated and developed. The primary goal of rehabilitation robots is to aid physiotherapists in the course of therapy by increasing quality and efficiency of the process. Effectiveness of using robots in rehabilitation has been shown with clinical results [1-3]. There are two main classifications of a rehabilitation program in general: therapeutic modalities and therapeutic exercises. While the goal of therapeutic modalities is to remove the effects of pain, spasm and edema, the ultimate aim of therapeutic exercises is to return injured patient to pre-injured healthy conditions and movement capabilities. In order to achieve complete treatment of patients, parameters that are given in a proper sequence below must be improved [4].

- Flexibility and range of motion.

- Strength and muscle endurance.

- Proprioception, coordination and agility.

There is a hierarchical order between these parameters, in which a preceding step is an obligatory or a conditional step for passing to the next. As can be seen from these parameters, a through rehabilitation program begins with a passive range of motion followed by assistive exercises and continues with resistive ones. First, passive range of motion exercises are usually recommended for patients in order to regain flexibility and wide range of motion in their muscles. Then, resistive and strength exercises have to be performed for recovering to pre-injured healthy conditions such as proprioception, coordination and agility. These steps are normally done under responsibility of a

\# This paper has been presented at the International Conference on Advanced Technology\&Sciences (ICAT'14) held in Antalya (Turkey), August 12-15, 2014.

*Corresponding Author: Email: bariscanyalcin@gmail.com physiotherapist. In addition, transportation of patients to a hospital or calling a physiotherapist to a place where the patient is located consumes time and results in high costs. Considering such intricacies of a rehabilitation process, treatment becomes more complex due to economic and social constraints. Engineers working in the field of robotics focus on designing novel rehabilitation robots that can make a difference to deal with these difficulties. From the engineering point of view, applications of robotic technologies are suitable to solve these problems due to the following reasons [5] :

- Robots are excellent mechanisms that can achieve repetitive movements in pre-determined frequencies.

- Robotic mechanisms are easy to control under variable forces.

- $\quad$ Robots can produce required forces during the process of interaction between human and machine.

Especially over the last fifteen years, many improvements have been achieved in rehabilitation robotic systems that can be used to satisfy expectations of patients. These systems have been designed to perform some repetitive, resistive and assistive exercises. An experimental 4-DOF system was built by Homma et al. [3] to carry out hip joint flexion/extension, hip joint abduction/adduction, hip joint external/internal rotation and knee joint flexion/extension motions. This system, which used a parallel wire mechanism, can be located around patient's bed. The purpose of using a wire mechanism instead of a rigid link was to reduce necessary motor power due to safety concerns. A 2-DOF autonomous system called NeXOS, which is able to perform passive, active and resistive exercises at patient's home environment was developed by Bradley et al. [6]. The aim of the study was to integrate home based and external services using internet. For example, it was possible to adjust the number of repetitions remotely. Moughamir et al. designed a training system for lower limbs called Multi-Iso [7]. The working principle of this system was based on switching control structures corresponding to required training programme (isokinetic, steering, isometric, isotonic, physiokinetic, stretching, and assisted). Some of these motions were originally developed for Multi-Iso machine. These specific motions defined by a 
physiotherapist were translated into force and position control laws using a man-machine interface. This system was able to perform passive, assistive and resistive exercises like NeXOS and used for knee limb extension - flexion movements. In the works mentioned above, the proposed control methods have lacked fully modeling physiotherapist motions due to absence of impedance parameters. Hogan et al. controlled SCARA named MANUS [8] that can provide two translational degrees of freedom for the forearm and elbow motion for rehabilitation purpose. The design was based on determining range of stiffness, force and impedance parameters at the end-effector. It is one of the most known systems among rehabilitation robotic systems and showed that impedance control method based on response of patients is a need in rehabilitation systems, also proved the impedance control is the most appropriate control method for a robot interacting with its environment. Bernhardt et al. designed LOKOMAT [2] that enabled the patient accomplish free walking motion using adaptive and impedance control algorithms. The adaptive algorithm was used to adapt the reference gait pattern by minimizing the interaction torques between patient and robot, thus this change allowed the patient to influence the gait pattern. The impedance algorithm provided an adjustable virtual springdamper element for pushing the patient's leg back to allow the patient to deviate from the reference gait pattern. TEM (therapeutic exercise machine) was developed by Okada et al. [9]. It works for helping patients who had stroke to recover their walking function using kinetic therapy on lower limbs. TEM has a function to imitate motions of physiotherapist that is not possible for conventional therapy machines. To imitate corresponding motions, impedance control method used in a 2DOF robotic system. Akdoğan et al. designed a therapeutic lower limb exercise robot named Physiotherabot [10] that is not only able to perform all active and resistive exercises, but also manual exercises of a physiotherapist. What distinguishes Physiotherabot from other equivalent systems is that it can perform abductionadduction movements of the hip and flexion-extension movements of the knee and the hip using impedance control method. The proposed control method in our study will be tested using Physiotherabot in future works.

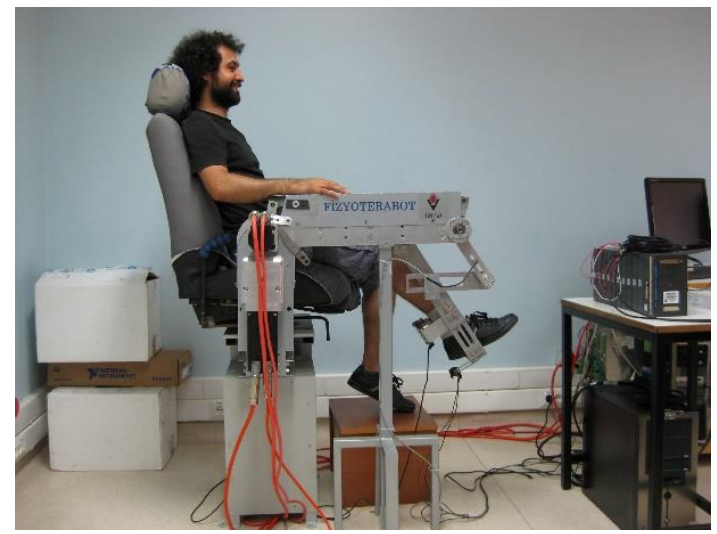

Figure 1. Physiotherabot.

Estimation of the impedance parameters that occur during patient-physiotherapist interaction plays an important role that provides smoothness and stability of motion during patient-robot interaction. Because of this reason, the impedance parameters of physiotherapist's arm have to be well estimated to achieve cooperative task between patient and robot. The impedance parameters of human arm change in different loading situations. Since complete definition of this variation was lacking, there was a need to know impedance parameters that occur during the contact between patient and physiotherapist. Several methods have been developed to estimate these parameters. Tsumugiwa $e t$ al. proposed a variable impedance control method for humanrobot interaction systems to maintain stability [11]. In this study, it was proved that a design that has dynamic parameters can be more suitable to deal with changing conditions, such as varying arm stiffness of a human during movement. Therefore, impedance parameters of robot arm were adjusted based on estimated human arm stiffness. Wang et al. proposed a fast online estimation method of impedance parameters based on the forgetting factor recursive least squares identification for robot control [12]. The proposed method performed fast tracking to parameter changes in different environments while achieving robustness to noise. Lakatos et al. modeled a neuromusculoskeletal system of a human arm as a rigid multibody system which consists of two bodies (the upper arm, the forearm) and considered muscles as force elements actuating the bodies [13], a general algorithm for optimally estimating impedance parameters of human arm was investigated.

In this work, we propose to teach a rehabilitation robot how to model passive flexion-extension movements performed by a physiotherapist for knee. A variable impedance control is proposed to teach optimum impedance parameters for a suitable exercise. This method causes the robot and a lower limb on it to follow flexion-extension motion determined by the physiotherapist, thus the most appropriate impedance parameters of the robot can be obtained for exercise. The variable impedance control method is divided into two steps. In the first step, learning phase, we investigate impedance characteristics of the physiotherapist's arm during the treatment in order to generate the impedance parameters of the robot. In the second step, treatment phase, the impedance of the robot obtained in the first step is utilized to emulate a physiotherapist's arm effect, as if a physiotherapist is applying it on lower limb of a patient.

This article proposes to address following function in rehabilitation robots: replicating and automating passive exercise motions normally applied by a physiotherapist for lower limb knee rehabilitation using variable impedance control method, as if a physiotherapist is applying them to a patient but only achieved by the therapy robot. The results show that modeling physiotherapist's arm as a single spring is sufficient and greatly simplifies the model.

The working principles of the robot are presented in section II. Results of the simulations are given in section III and the conclusions are found in section IV.

\section{Working Principle of the Robot}

\subsection{Learning Phase}

The proposed control method is based on adjusting impedance parameters, namely inertia and torsional damping coefficients of the robot, in terms of estimated stiffness coefficient of the physiotherapist's arm. We assume that the robot has only $I_{R}(t)$ inertia and $B_{R}(t)$ torsional damping impedance coefficient. $F_{P}(t)$ is the force that occurs due to robot-physiotherapist interaction, $T_{P}(t)$ is the torque that occurred by $F_{P}(t)$ at $\mathrm{O}$ point of the robot, $\theta_{D}(t)$ is desired angular position of the robot, because this position data is obtained when the robot is actuated with optimum impedance coefficients. Therefore, the mathematical model of the 1-DOF robot can be written in terms of joint space variables in (1).

$T_{P}(t)=I_{R}(t) \ddot{\theta}_{D}(t)+B_{R}(t) \dot{\theta}_{D}(t)$ 
and the mathematical model of the physiotherapist's arm can be written in terms of workspace variables in (2), physiotherapist's arm has only $K_{P}(t)$ stiffness coefficient. $X(t)$ is position difference, which is along physiotherapist's arm.

$$
F_{P}(t)=K_{P}(t) X(t)
$$

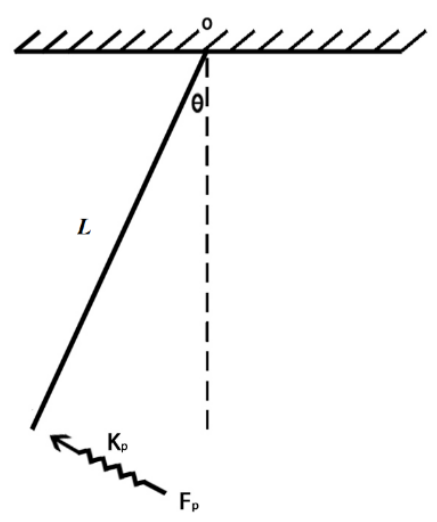

Figure 2. The robot modeled as a simple pendulum and physiotherapist's force on it.

As can be seen from Fig. 2, $L$ is the length between end-effector and point $\mathrm{O}$.

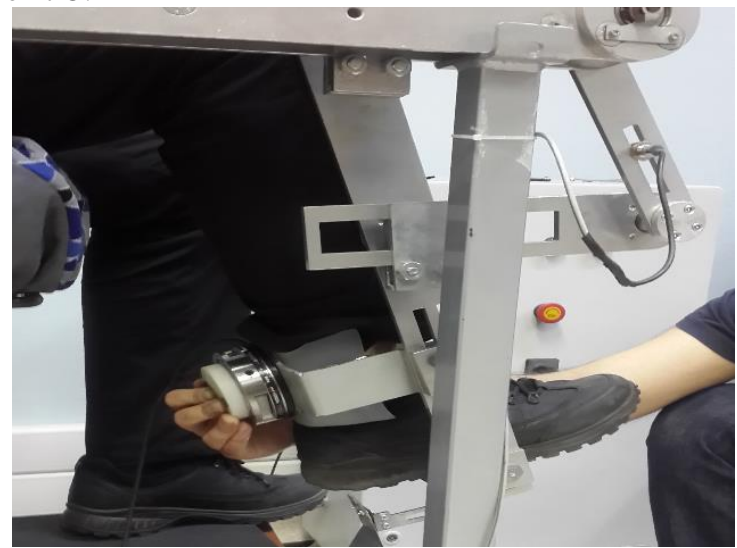

Figure 3. Physiotherapist's hand on the Physiotherabot.

$\theta_{R}(t)$ is recorded angular position of a patient's knee and $F_{P}(t)$ is recorded force data during an exercise applied by a real physiotherapist, and these data are taken from [14]. As can be seen from Fig.4, we assume that $K_{P}(t)$, the stiffness of physiotherapist's arm can be thought as $K_{O}(t)$, the robot's torsional stiffness that occurs at point $\mathrm{O}$ of the robot using (3), (4), (5), (6) and (7).

$$
\begin{aligned}
& X(t)=L \theta_{R}(t) \\
& F_{P}(t)=K_{P}(t) L \theta_{R}(t) \\
& F_{P}(t) L=T_{P}(t) \\
& T_{P}(t)=K_{O}(t) \theta_{R}(t)
\end{aligned}
$$

Thus;

$$
K_{P}(t) L^{2}=K_{O}(t)
$$

When we estimate the torsional stiffness of the robot, we will be able to generate appropriate impedance parameters such as inertia
$I_{R}(t)$ and torsional damping $B_{R}(t)$ to smoothly follow the intended motion of the physiotherapist using proportional coefficients $\alpha$ and $\beta$. We calculate $I_{R}(t)$ and $B_{R}(t)$ impedance parameters in the proportion of positive real numbers $\alpha, \beta$ and the absolute value of the estimated stiffness $K_{O}(t)$ as follows.

$$
\begin{aligned}
& I_{R}(t)=\alpha\left|K_{O}(t)\right| \\
& B_{R}(t)=\beta\left|K_{O}(t)\right|
\end{aligned}
$$

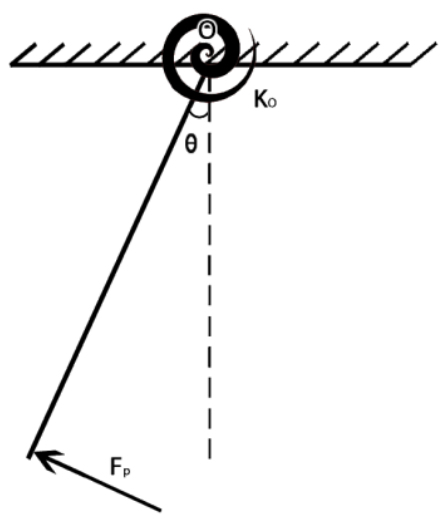

Figure 4. Torsional stiffness on the robot.

In this phase, the robot has learned the appropriate impedance parameters $I_{R}(t)$ and $B_{R}(t)$ with respect to the estimated stiffness parameter of the physiotherapist's arm. These impedance parameters will be used to generate the appropriate trajectory $\theta_{D}(t)$ to follow $\theta_{R}(t)$ in treatment phase.

\subsection{Treatment Phase}

In this phase, the impedance parameters of the robot obtained in learning phase will enable us to determine the most appropriate angular position pattern $\theta_{D}(t)$, as if a physiotherapist is applying the motion on lower limb of a patient. From (1), (8) and (9), we derive $\theta_{D}(t)$ parameter that provides the appropriate motion pattern for knee rehabilitation. Controller input to actuator and output torque which actuator produces for following $\theta_{D}(t)$ are not the subjects of this study, it is only determined the most appropriate $\theta_{D}(t)$ pattern for the patient according to estimated impedance parameters, $F_{P}(t) \square$ and $\theta_{R}(t)$ data are taken from [14] and shown in Fig.6. These data are used in (4) to estimate $K_{O}(t)$ parameter. 


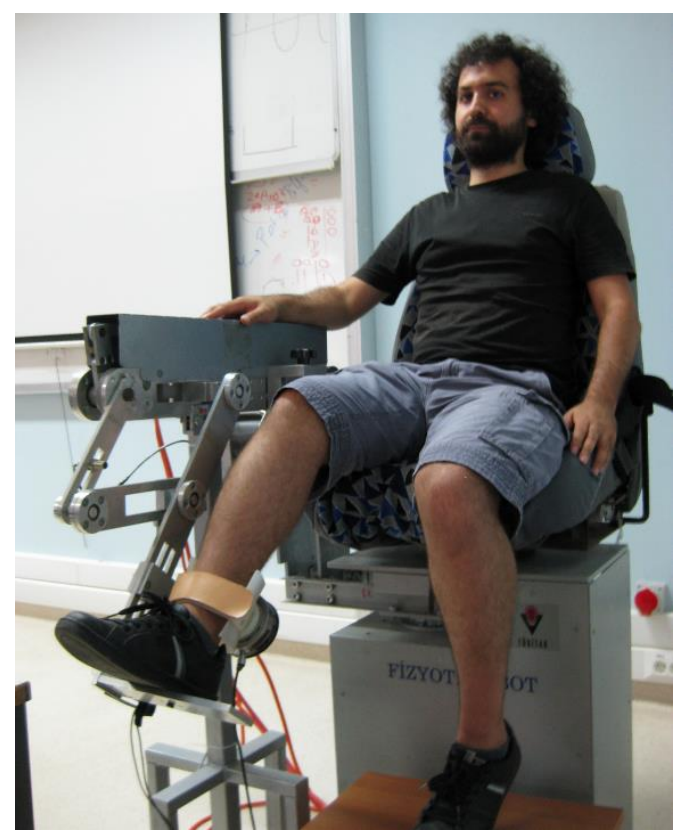

Figure 5. Treatment phase.

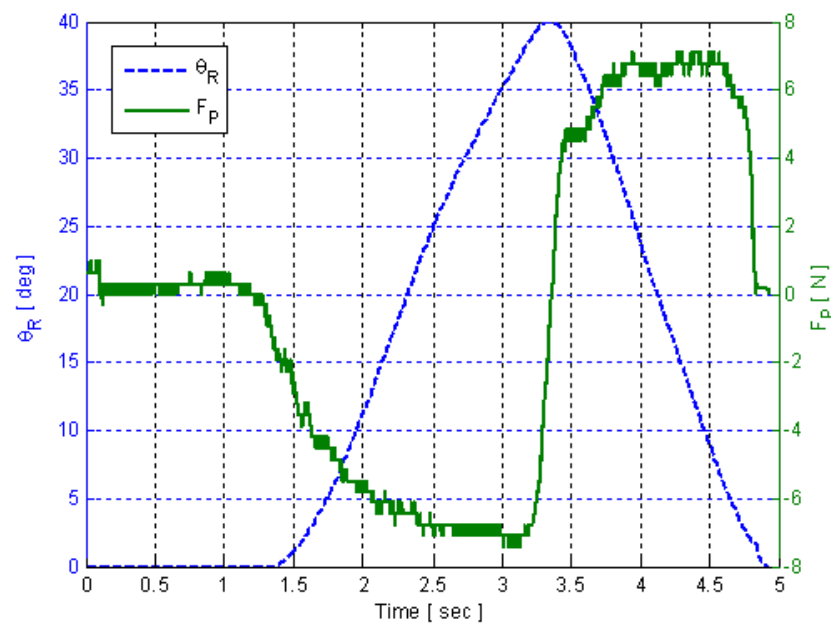

Figure 6. Real $F_{P}(t)$ and $\theta_{R}(t)$ data.

Using (7), absolute value of $K_{O}(t)$ shown in Fig.7 is calculated, and impedance parameters $I_{R}(t)$ and $B_{R}(t)$ will be used to determine $\theta_{D}(t)$ pattern to model physiotherapist's motion using (1), an example can be seen in Fig.8 for parameters $\alpha=0.1$ and $\beta=3$.

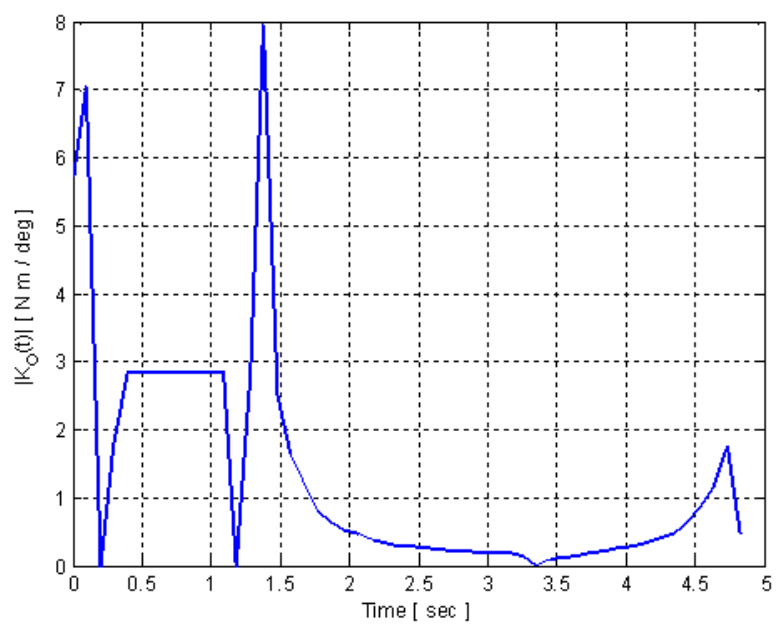

Figure 7. $\left|K_{O}(t)\right|$ parameter.

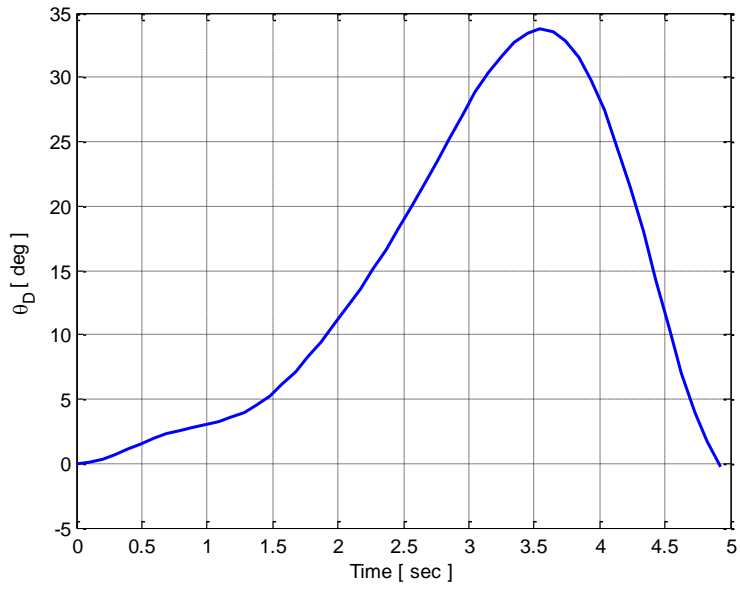

Figure 8. $\theta_{D}(t)$ parameter for $\alpha=0.1 \beta=3$.

\section{Simulation Results}

In this section, several $\alpha$ and $\beta$ values we choose are tested to obtain the most appropriate $\theta_{D}(t)$ pattern which follows $\theta_{R}(t)$, $\theta_{D}(t)$ patterns determined by different $\alpha$ and $\beta$ values as can be seen in Fig.9 and errors are shown in Fig. 10. The values which can locate the error as possible as near zero will be used for experimental verification of simulation using Physiotherabot in future works.

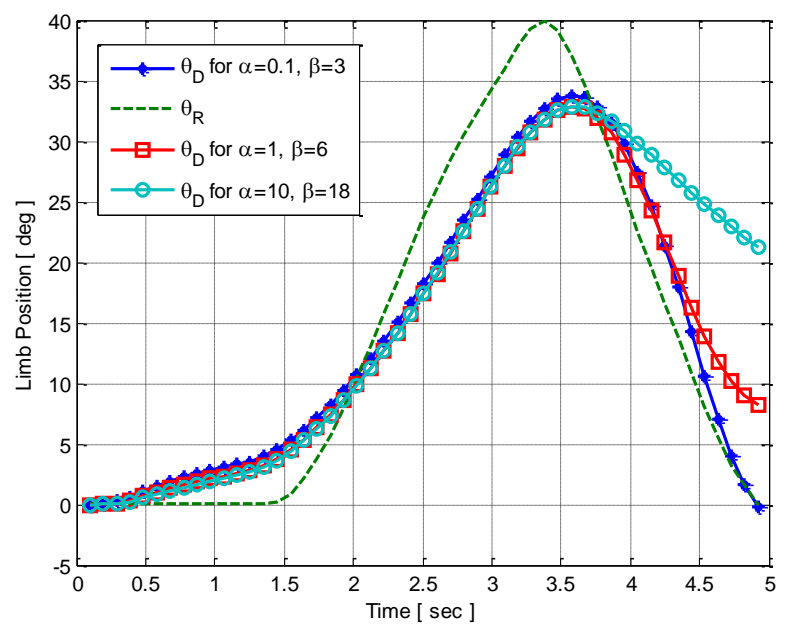

Figure 9. $\theta_{D}(t)$ and $\theta_{R}(t)$ parameter for $\alpha=0.1 \beta=3, \alpha=1 \beta=6$ and $\alpha$ $=10 \beta=18$

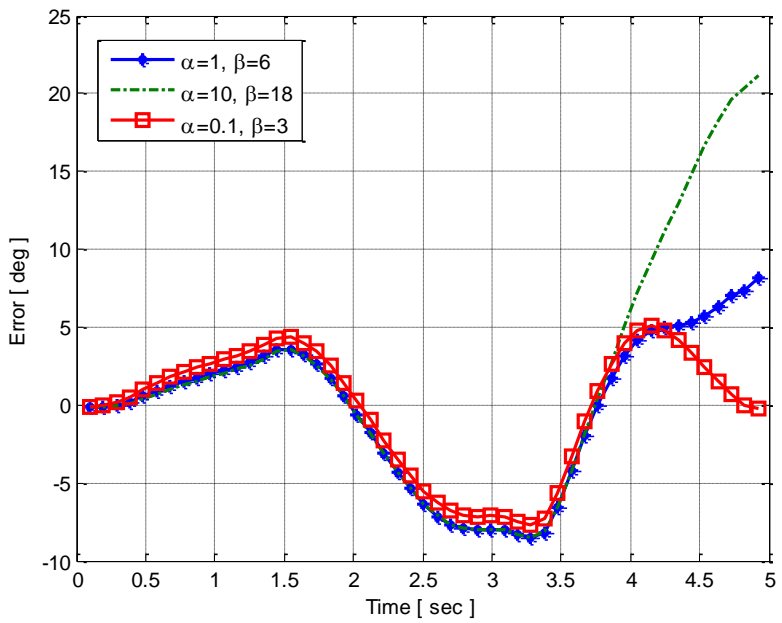

Figure 10. Error between $\theta_{D}(t)$ and $\theta_{R}(t)$ parameters for $\alpha=0.1 \beta=3, \alpha$ $=1 \beta=6$ and $\alpha=10 \beta=18$. 


\section{Conclusion}

Simulation results show that adjusting impedance parameters that are in terms of estimated stiffness parameter of the physiotherapist's arm enables us to create a physiotherapist effect for passive lower limb flexion-extension exercises. This method gives us an error $\left(\max .7^{\circ}\right.$ for parameters $\alpha=0.1, \beta=3$ ) which is acceptable for passive flexion-extension movements for lower limb rehabilitation as can be seen in Fig.10, thus we can say that modeling physiotherapist's arm as a single spring is sufficient at least for passive flexion-extension exercises in lower limbs.

Future work includes experimental verification of the findings presented in this paper using Physiotherabot. A more sophisticated model for estimating $\alpha$ and $\beta$ parameters can be based on Lyapunov approach and some identical parameters of patient, such as lower limb's mass and inertia can be used as a controller parameter in direct or indirect model reference adaptive control schemes. In addition to the single spring model, physiotherapist's arm can be modeled as a mass-damper-spring model to have more precise results.

\section{References}

[1] A. Toth, G. Fazekas, G. Arz, M. Jurak, and M. Horvath, "Passive robotic movement therapy of the spastic hemiparetic arm with REHAROB: report of the first clinical test and the follow-up system improvement," in Rehabilitation Robotics, 2005. ICORR 2005. 9th International Conference on, 2005, pp. 127-130.

[2] M. Bernhardt, M. Frey, G. Colombo, and R. Riener, "Hybrid force-position control yields cooperative behaviour of the rehabilitation robot LOKOMAT," in Rehabilitation Robotics, 2005. ICORR 2005. 9th International Conference on, 2005, pp. 536-539.

[3] K. Homma, O. Fukuda, Y. Nagata, and M. Usuba, "Study of a wire-driven leg rehabilitation system," in Intelligent Robots and Systems, 2004. (IROS 2004). Proceedings. 2004 IEEE/RSJ International Conference on, 2004, pp. 16681673 vol.2.

[4] H. PA, Therapeutic exercises for musculoskeletal injuries, 2009.

[5] H. I. Krebs, "An overwiev of rehabilitation robotic technologies. In: American spinal injury association symposium," 2006.

[6] D. A. Bradley, C. Acosta-Marquez, M. Hawley, S. Brownsell, P. Enderby, and S. Mawson, "Remote rehabilitation - The NeXOS project: Lessons learnt and questions raised," in Rehabilitation Robotics, 2009. ICORR 2009. IEEE International Conference on, 2009, pp. 956961.

[7] S. Moughamir, N. Manamanni, J. Zaytoon, and L. Afilal, "Control law implementation for Multi-Iso: a training machine for lower limbs," in Engineering in Medicine and Biology Society, 2001. Proceedings of the 23rd Annual International Conference of the IEEE, 2001, pp. 1477-1480 vol.2.

[8] N. Hogan, H. I. Krebs, J. Charnnarong, P. Srikrishna, and A. Sharon, "MIT-MANUS: a workstation for manual therapy and training. I," in Robot and Human Communication, 1992. Proceedings., IEEE International Workshop on, 1992, pp. 161-165.

[9] S. Okada, T. Sakaki, R. Hirata, Y. Okajima, S. Uchida, and Y. Tomita, "TEM: a therapeutic exercise machine for the lower extremities of spastic patients," Advanced Robotics, vol. 14, pp. 597-606, 2001/01/01 2001.

[10] E. Akdogan and M. A. Adli, "The design and control of a therapeutic exercise robot for lower limb rehabilitation: Physiotherabot," Mechatronics, vol. 21, pp. 509-522, 2011.

[11] T. Tsumugiwa, R. Yokogawa, and K. Hara, "Variable impedance control based on estimation of human arm stiffness for human-robot cooperative calligraphic task," in Robotics and Automation, 2002. Proceedings. ICRA '02. IEEE International Conference on, 2002, pp. 644-650 vol.1.

[12] W. Zheng, A. Peer, and M. Buss, "Fast online impedance estimation for robot control," in Mechatronics, 2009. ICM 2009. IEEE International Conference on, 2009, pp. 1-6.

[13] D. Lakatos, F. Petit, and P. van der Smagt, "Conditioning vs. excitation time for estimating impedance parameters of the human arm," in Humanoid Robots (Humanoids), 2011 11th IEEE-RAS International Conference on, 2011, pp. 636-642.

[14] E. Akdogan, "Intelligent Position and Force Control of A Robot Manipulator for Rehabilitation," Institute for Graduate Studies in Pure and Applied Sciences, Marmara University, 2007. 